\title{
Genes identified in Asian SLE GWASs are also associated with SLE in Caucasian populations
}

\author{
Chuan Wang' ${ }^{1}$ Annika Ahlford ${ }^{1,16}$, Tiina M Järvinen ${ }^{2,17}$, Gunnel Nordmark ${ }^{3}$, Maija-Leena Eloranta ${ }^{3}$, \\ Iva Gunnarsson ${ }^{4}$, Elisabet Svenungsson ${ }^{4}$, Leonid Padyukov ${ }^{4}$, Gunnar Sturfelt ${ }^{5}$, Andreas Jönsen ${ }^{5}$, \\ Anders A Bengtsson ${ }^{5}$, Lennart Truedsson ${ }^{6}$, Catharina Eriksson ${ }^{7}$, Solbritt Rantapää-Dahlqvist ${ }^{8}$, Christopher \\ Sjöwall ${ }^{9}$, Heikki Julkunen ${ }^{10}$, Lindsey A Criswell ${ }^{11}$, Robert R Graham ${ }^{12}$, Timothy W Behrens ${ }^{12}$, Juha Kere ${ }^{2,13,14}$, \\ Lars Rönnblom ${ }^{3}$, Ann-Christine Syvänen ${ }^{1}$ and Johanna K Sandling ${ }^{\star, 1,15}$
}

Recent genome-wide association studies (GWASs) conducted in Asian populations have identified novel risk loci for systemic lupus erythematosus (SLE). Here, we genotyped 10 single-nucleotide polymorphisms (SNPs) in eight such loci and investigated their disease associations in three independent Caucasian SLE case-control cohorts recruited from Sweden, Finland and the United States. The disease associations of the SNPs in ETS1, IKZF1, LRRC18-WDFY4, RASGRP3, SLC15A4, TNIP1 and $16 p 11.2$ were replicated, whereas no solid evidence of association was observed for the $7 q 11.23$ locus in the Caucasian cohorts. SLC15A4 was significantly associated with renal involvement in SLE. The association of TNIP1 was more pronounced in SLE patients with renal and immunological disorder, which is corroborated by two previous studies in Asian cohorts. The effects of all the associated SNPs, either conferring risk for or being protective against SLE, were in the same direction in Caucasians and Asians. The magnitudes of the allelic effects for most of the SNPs were also comparable across different ethnic groups. On the contrary, remarkable differences in allele frequencies between Caucasian and Asian populations were observed for all associated SNPs. In conclusion, most of the novel SLE risk loci identified by GWASs in Asian populations were also associated with SLE in Caucasian populations. We observed both similarities and differences with respect to the effect sizes and risk allele frequencies across ethnicities.

European Journal of Human Genetics (2013) 21, 994-999; doi:10.1038/ejhg.2012.277; published online 19 December 2012

Keywords: systemic lupus erythematosus; genetic-association study; Asian; Caucasian

\section{INTRODUCTION}

Systemic lupus erythematosus (SLE) is an autoimmune disorder with a heterogeneous clinical spectrum, extending from mild cases limited to skin and joint manifestations to life-threatening conditions with renal impairment, severe cytopenias, central nervous system disease or thromboembolic events. The contribution of heritable factors to SLE predisposition is evident from the strong familial aggregation and high disease concordance in monozygotic twins $(24-56 \%) .{ }^{1}$ Since $2008,>25$ potential risk loci for SLE have been identified in genomewide association studies (GWASs) in Caucasian populations. ${ }^{2-6}$ These risk alleles account for about $8-15 \%$ of the total genetic susceptibility to the disease. ${ }^{7,8}$

Shortly after the findings of risk alleles for SLE in Caucasians, GWASs with similar or larger sample sizes were conducted in East
Asian populations. ${ }^{9,10}$ These studies not only confirmed the association signals for some of the major genetic loci reported in Caucasians, including BLK, IRF5, STAT4, TNFAIP3, TNFSF4 and the $H L A$ region, but also discovered novel SLE risk loci. Two of the novel SLE genes, TNIP1 and IKZF1, were independently identified in Caucasian $^{7,11}$ and Asian $^{9}$ populations, suggesting that the majority of genetic risk factors for SLE are common across Caucasians and Asians. However, a higher proportion of SLE risk loci seem to be shared between different Asian populations compared with the loci they share with Caucasian populations. ${ }^{12}$ In addition, higher incidence and prevalence of SLE, as well as differences in clinical manifestations have been reported for Asian populations. ${ }^{13}$ These observations indicate potential genetic heterogeneity for SLE between Asian and Caucasian populations.

${ }^{1}$ Department of Medical Sciences, Molecular Medicine and Science for Life Laboratory, Uppsala University, Uppsala, Sweden; ${ }^{2}$ Department of Medical Genetics, Haartman Institute, and Molecular Medicine Research Program, Research Programs Unit, University of Helsinki, Helsinki, Finland; ${ }^{3}$ Department of Medical Sciences, Rheumatology, Uppsala University, Uppsala, Sweden; ${ }^{4}$ Rheumatology Unit, Department of Medicine, Karolinska University Hospital, and Karolinska Institutet, Stockholm, Sweden; ${ }^{5}$ Department of Clinical Sciences, Section of Rheumatology, Lund University, Lund, Sweden; ${ }^{5}$ Section of MIG, Department of Laboratory Medicine, Lund University, Lund, Sweden; ${ }^{7}$ Department of Clinical Immunology, Umeå University Hospital, Umeå, Sweden; ${ }^{8}$ Department of Public Health and Clinical Medicine, Rheumatology, Umeå University, Umeå, Sweden; ${ }^{9}$ Rheumatology/AIR, Department of Clinical and Experimental Medicine, Linköping University, Linköping, Sweden; ${ }^{10}$ Department of Rheumatology, Helsinki University Central Hospital, Peijas Hospital, Vantaa, Finland; ${ }^{11}$ Rosalind Russell Medical Research Center for Arthritis, Department of Medicine, University of California, San Francisco, CA, USA; ${ }^{12}$ Human Genetics Group, Genentech Inc., South San Francisco, CA, USA; ${ }^{13}$ Folkhälsan Institute of Genetics, Helsinki, Finland; ${ }^{14}$ Department of Biosciences and Nutrition, Karolinska Institutet, Huddinge, Sweden; ${ }^{15}$ Department of Human Genetics, Genetics of Complex Traits in Humans, Wellcome Trust Sanger Institute, Hinxton, Cambridge, UK 16Present address: Dr A Ahlford, Science for Life Laboratory, Department of Biochemistry and Biophysics, Molecular Diagnostics, Stockholm University, Sweden

${ }^{17}$ Present address: Dr TM Järvinen, Department of Molecular Virology, Immunology and Medical Genetics, The Ohio State University Comprehensive Cancer Center, Columbus, $\mathrm{OH}, \mathrm{USA}$

*Correspondence: Dr JK Sandling, Genetics of Complex Traits in Humans, Department of Human Genetics, Wellcome Trust Sanger Institute, Hinxton, Cambridge CB10 1SA, UK Tel: +44 1223 494838; Fax: +44 1223 494919; Email: js24@sanger.ac.uk

Received 10 July 2012; revised 13 November 2012; accepted 15 November 2012; published online 19 December 2012 
In this study, we investigated whether 10 single-nucleotide polymorphisms (SNPs) located at eight SLE risk loci, including ETS1, IKZF1, LRRC18-WDFY4, RASGRP3, SLC15A4, TNIP1, 7q11.23 and 16p11.2, which were originally identified by GWAS in Asian populations, ${ }^{9}$ also confer disease risk in a collection of Caucasian SLE patients and healthy controls from Sweden and Finland. We also present data for these loci from our previous study on Caucasians from the United States.

\section{MATERIALS AND METHODS}

\section{Subjects}

The Swedish cohort included 1129 SLE patients and 2060 controls. Patients were recruited from the Rheumatology clinics at the Uppsala, Karolinska (Solna), Umeå, Lund and Linköping University Hospitals, and the controls were either healthy blood donors (Uppsala, Umeå, Lund and Linköping) or controls from the population-based Epidemiological Investigation of Rheumatoid Arthritis cohort. ${ }^{14}$ Detailed clinical characteristics were available for 873 Swedish SLE patients (Supplementary Table S1). The Finnish cohort was composed of 270 SLE patients recruited from the Helsinki, Tampere and Kuopio University Hospitals and 343 controls from multiple regions in Finland. ${ }^{15,16}$ The US cohort, consisting of 1310 SLE patients and 7859 controls has been previously described. ${ }^{3,7}$ All participants included in the current study were of Caucasian origin based on self-report. All SLE patients fulfilled at least four of the classification criteria for SLE as defined by the American College of Rheumatology (ACR). ${ }^{17}$ The study was approved by the ethics committees of all involved institutions. Informed consents were obtained from all SLE patients and controls.

\section{Genotyping}

Ten novel polymorphisms from a SLE GWAS in Han Chinese ${ }^{9}$ were selected for genotyping. One additional SNP (rs7708392, TNIP1) was selected from a previous study on Caucasians. ${ }^{7}$ Individuals from the Swedish and Finnish SLE case-control cohorts were both genotyped for the eleven SNPs with a 384-plex GoldenGate assay using VeraCode microbeads on the Illumina BeadXpress system (Illumina Inc, San Diego, CA, USA) at the SNP and SEQ technology platform in Uppsala, Sweden (www.genotyping.se). One SNP failed genotype clustering (rs4639966, 11q23.3). Genotype data for the 10 remaining SNPs were extracted from the larger set of $384 \mathrm{SNPs}$, and the data for both cohorts were then quality controlled together. A genotyping success rate of $95-97 \%$ was achieved for all 10 SNPs. Call rates for the Illumina GoldenGate assay will largely depend on the SNPs in the oligo pool. Our 384-plex SNP panel included a mix of novel (not previously validated) and common variants which could have contributed to the observed slightly lower call rates. In total 106 individuals with more than two failed SNPs were excluded from subsequent analyses. All 10 SNPs were in Hardy-Weinberg equilibrium $(P$-value $>0.005)$ in the control individuals from both cohorts. For the US SLE case-control cohort, quality controlled genotype data for 8 out of the 10 SNPs was available from a previous study by Gateva et al. ${ }^{7}$ The 10 SNPs genotyped and analyzed in the Swedish and Finnish cohorts in the current study were selected independently of what data that was available for the US cohort.

\section{Statistical analysis}

Quality control of the genotype data, Hardy-Weinberg equilibrium tests, and association analyses were conducted using the software PLINK. ${ }^{18}$ We performed allelic association analysis using Fisher's exact tests by comparing the allele counts of SNPs in SLE patients and controls, in case numbers in any of the groups would be so small that the $\chi^{2}$-approximation would be invalid. Odds ratios (ORs) were reported relative to the risk allele identified in Asians. Similarly, subphenotype case-only analyses were performed by comparing allele counts for the patients who fulfilled a specific ACR classification criterion for SLE to all the SLE patients who did not fulfill that specific criterion. Subphenotype case-control analyses were performed by comparing allele counts for the patients who fulfilled a specific ACR classification criterion for SLE to healthy controls. A $P$-value $\leqslant 0.05$ was considered significant. Heterogeneity of allelic effects across cohorts was evaluated with Cochran's
Q-test and the $I^{2}$ heterogeneity index. ${ }^{19}$ Meta-analysis was carried out using the software GWAMA v2.0.7, ${ }^{20}$ in which a fixed-effects model was used for SNPs with a $P$-value $>0.05$ in Cochran's $Q$-test and a random-effects model for SNPs with a $P$-value $\leqslant 0.05$. For rs7708392 and rs4917014 meta-analysis was conducted based on the data from the Swedish and the Finnish cohorts, and for all other SNPs data from all three cohorts were included. Population attributable risk percentage (PAR\%), which considers both the OR and risk allele frequency (RAF) in the general population, was estimated using the formula $\mathrm{RAF}(\mathrm{OR}-1) /(\mathrm{RAF}(\mathrm{OR}-1)+1){ }^{21}$ Statistical power was estimated using the software Quanto v1.2.4 (http://hydra.usc.edu/gxe/) with a log-additive model, a two-sided type I error rate of 0.05 and SLE prevalence of $0.068 \%$ in Sweden, $0.028 \%$ in Finland and $0.053 \%$ in the United States. ${ }^{22}$

\section{RESULTS}

\section{Allelic association analysis in three Caucasian SLE case-control} cohorts

Ten SNPs in nine novel SLE loci discovered in Asian populations, ${ }^{9}$ but which had not yet been replicated in Caucasians, were selected for inclusion in the study. These loci were ETS1, IKZF1, LRRC18WDFY4, RASGRP3, SLC15A4, TNIP1, 7q11.23, 11q23.3 and 16p11.2. A SNP in TNIP1 (rs7708392) from our previous study on Caucasians published at the same time was also included, as this SNP differed from the one reported in Asians. ${ }^{7}$ We genotyped and investigated the disease associations for these SNPs in two Caucasian SLE case-control cohorts from Sweden and Finland. Data for these loci were then extracted from our previous study on Caucasians from the United States. In the Swedish, Finnish, and US SLE case-control cohorts, consistent and significant association signals were detected for the SNPs rs1913517 and rs6590330, which are annotated to the gene loci LRRC18-WDFY4 and ETS1, respectively, (Table 1, Supplementary Table S2). The associations with SLE for the TNIP1 (encoding TNFAIP3-interacting protein 1) and SLC15A4 (solute-carrier family 15, member 4) genes were supported by data from two cohorts: TNIP1 (rs10036748) in the Swedish and US cohorts, and SLC15A4 (rs10847697 and rs1385374) in the Swedish and Finnish cohorts. In the remaining third cohort the association was in the same direction as in the other two but with marginally significant $P$-values. The SNPs tagging the RASGRP3 (RAS guanyl nucleotide-releasing protein 3) and $7 q 11.23$ loci appeared to be associated with SLE in the Swedish cohort, but failed to replicate in the Finnish or the US cohort. IKZF1 (Ikaros family zinc finger 1) was also exclusively associated with SLE in the Swedish cohort, but no SNP tagging this gene was tested in the US cohort. Additionally, the SNP rs7197475, which is located in the 16p11.2 locus, showed a trend of association in the Swedish cohort only. The SNP rs4639966 in the 11q23.3 locus failed genotyping in the Swedish and Finnish cohorts. In the Swedish and the US cohorts, we have $>58 \%$ power with a hypothesized OR of 1.2 , and $>89 \%$ power with a hypothesized OR of 1.3 for the SNPs without nominally significant $P$-values; whereas the Finnish cohort is smaller in size and provides limited power (10-35\% and 16-62\% with a hypothesized OR of 1.2 and 1.3 , respectively).

In a meta-analysis significant $P$-values for the association with SLE were detected for all SNPs except rs1167796 at the 7q11.23 locus (Table 1). In addition, the effects of all the associated alleles, either conferring risk for SLE or being protective against SLE, were in the same direction as reported in the Asian cohort.

\section{Comparisons of allele frequencies, effect sizes and risk across cohorts}

In order to more comprehensively evaluate the differences in disease association for the SLE risk loci between Asian and Caucasian populations, data from the Swedish, Finnish and US cohorts in the 


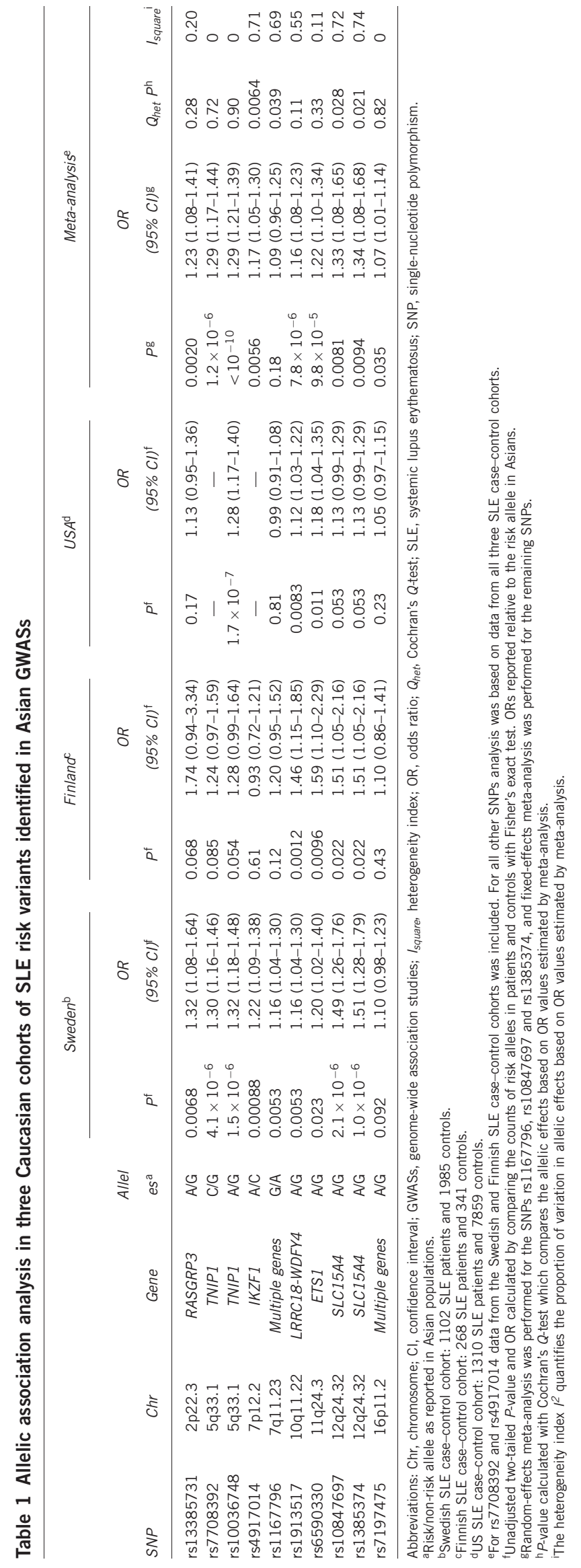

current study were analyzed as one combined Caucasian cohort. In addition, data on the TNIP1 SNP rs6889239, which is in perfect LD with rs10036748 tested here, from a published UK SLE case-control study was also included in this Caucasian cohort. ${ }^{11}$ Analogously, summary data were retrieved from three published Chinese SLE association studies ${ }^{9,10,23}$ and two published Japanese SLE association studies, ${ }^{12,24}$ and summarized into one Asian cohort.

The RAFs of all 10 SNPs in control individuals differed significantly between the combined data sets of Caucasians and Asians (Fisher's exact test $P$-value $<10^{-10}$, Table 2). Especially for the TNIP1 SNPs rs10036748 and rs7708392, and rs1913517 in LRRC18-WDFY4, their minor alleles in Caucasians were the major alleles in Asians (Figure 1). Despite the clear difference in allele frequencies for the SNPs between Caucasians and Asians, their effect sizes, which were estimated by ORs derived from meta-analysis, were not as distinct across populations (Table 2, Figure 1). The effect sizes of the SNPs rs6590330 and rs7197475 were significantly larger in the Asian population (Cochran's $Q$-test $P$-value $\left.\leqslant 0.05, I^{2} \geqslant 0.84\right)$, and moderate heterogeneity of allelic effect $\left(0.49 \leqslant I^{2} \leqslant 0.61\right)$ was detected for the SNPs rs13385731, rs10036748, rs4917014, rs1167796 and rs1913517. The contributions of all ten SNPs to the risk of SLE were estimated with PAR\%, which considers both RAF and effect size. Higher PAR\% values were detected for most of the SNPs in the Asian population (Table 2). Notably the PAR\% value of the ETS1 SNP rs6590330 was about five times higher in Asians than in Caucasians. For the SNPs rs7708392, rs10036748 and rs1167796, located in the TNIP1 and 7q11.23 loci, their risk contributions in Asians were more than double the size than in Caucasians.

The RAFs and effect sizes for the 10 SNPs were also compared across the Swedish, Finnish and US SLE case-control cohorts (Figure 1, Supplementary Table S2, Supplementary Table S3). Most of the SNPs showed some differences in RAFs across the three cohorts, although the differences were not as pronounced as compared with the Asian cohort. In contrast, the effect sizes of all SNPs were comparable in different Caucasian cohorts. Only marginally significant differences were detected for the SLC15A4 and the $7 q 11.23$ loci, which seemed to confer a stronger risk in the Swedish cohort than in the US cohort. PAR\% values across different Caucasian cohorts were also variable, which was mainly a consequence of the differences in allele frequencies.

\section{Association analysis within SLE patients with different disease manifestations}

Considering that the genetic risk factors for SLE influence different cellular pathways, their contribution to disease pathogenesis may differ across disease manifestations. Therefore, we performed caseonly association analysis in subgroups of Swedish SLE patients with ACR classification data available (Supplementary Tables S1, S4). Significant association signals were detected for the genes TNIP1 and SLC15A4 in the SLE patients with renal disorder (ACR7, Supplementary Table S4). Together with ETS1, TNIP1 seemed to also be associated with the immunological disorder criterion (ACR10). In addition, RASGRP3 showed association signals in the SLE patients with neurological disorder (ACR8), and 16p11.2 was associated with photosensitivity (ACR3) as well as serositis (ACR6). However, only the association for SLC15A4 in the SLE patients with renal disorder (ACR7) remained significant after multiple testing correction. We also performed a subphenotype case-control analysis, where we found TNIP1 and SLC15A4 to be associated with 8 of the 11 SLE subgroups after applying multiple testing correction (Supplementary Table S5). 
Table 2 Comparisons of RAFs, effect sizes and PAR\% between Caucasians and Asians

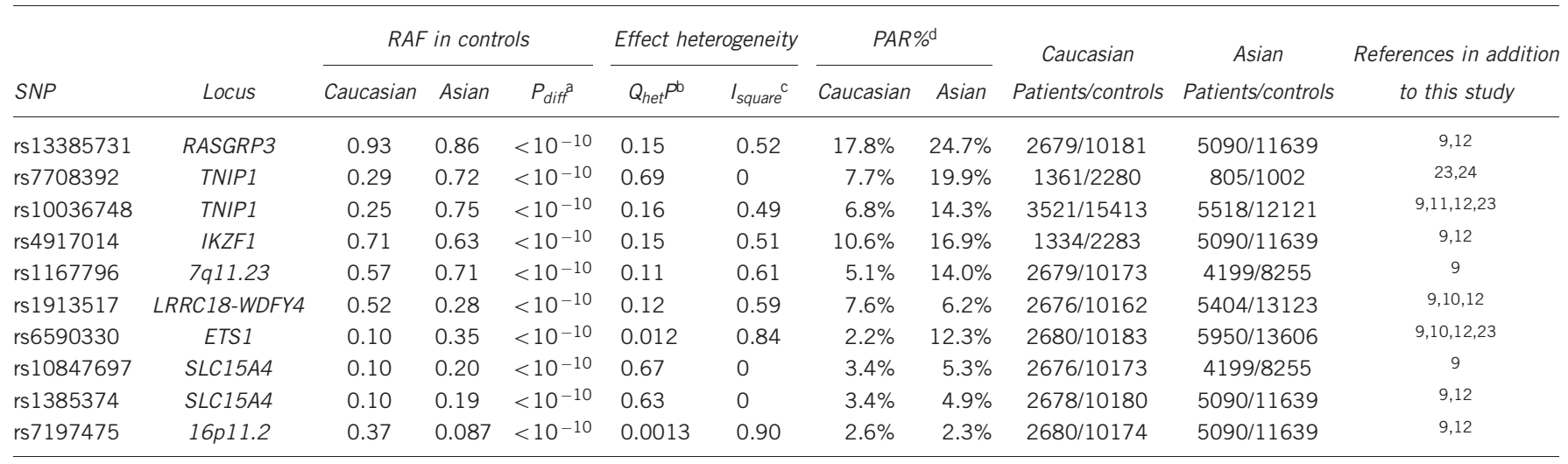

Abbreviations: $I_{\text {square, }}$, heterogeneity index; OR, odds ratio; PAR\%, population attributable risk percentage; $Q_{\text {het, }}$ Cochran's $Q$-test; RAF, risk allele frequency; SNP, single-nucleotide polymorphism. aTwo-tailed $P$-value calculated by comparing the counts of risk alleles in the combined Caucasian and combined Asian data sets of controls with Fisher's exact test.

b $P$-value calculated with Cochran's $Q$-test which compares the allelic effects based on OR values estimated by meta-analysis.

CThe heterogeneity index $R^{2}$ quantifies the proportion of variation in allelic effects based on OR values estimated by meta-analysis.

dPAR\% calculated using the formula RAF(OR-1)/(RAF[OR-1]+1) ${ }^{21}$

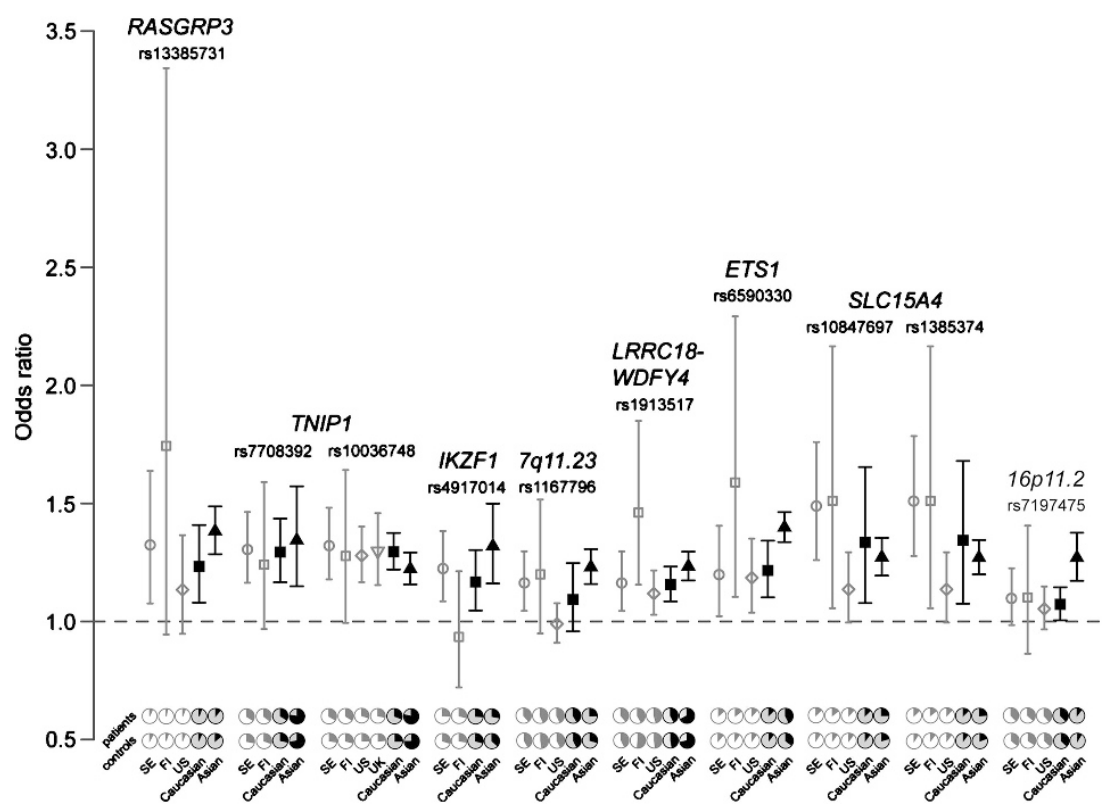

Figure 1 Comparisons of odds ratios (ORs) and allele frequencies across cohorts. The median of ORs for individual cohorts are plotted with open symbols in gray, and the counterparts estimated from meta-analysis in Caucasian and Asian populations are plotted with solid symbols in black. ORs are calculated based on the risk alleles of SNPs reported in Asian populations. Error bars represent 95\% confidence intervals (Cls). The pie charts at the bottom panel illustrate the allele frequencies of SNPs in patients and controls. Charts for the combined data sets of Caucasians and Asians are in a darker shade.

\section{DISCUSSION}

By investigating 10 SNPs in three independent Caucasian SLE casecontrol cohorts, we replicated the association signals from 9 out of the 10 SLE SNPs originally identified by GWASs in Asian SLE casecontrol cohorts. Applying the more stringent Bonferroni correction, five of these would still be significant. With the current study design, the statistical power for all the significant association signals was $>65 \%$ in the individual Caucasian cohorts and $>99 \%$ for the meta-analysis. Of the associated genes, both LRRC18 (leucine-rich repeat-containing protein 18) and WDFY4 (WD repeat and FYVE domain-containing protein 4) are of unknown function, whereas ETS1 encodes an ETS transcription factor involved in B-cell development, mutation of which leads to autoimmune disease in mice. $^{25}$ TNIP1 is a regulatory protein that inhibits signaling by several receptors, including TNF-alpha, TLR and PPAR. ${ }^{26}$ SLC15A4 is highly expressed in antigen-presenting cells, and has been shown to regulate TLR9- and NOD1-dependent innate immunity responses. ${ }^{25}$ The IKZF1 transcription factor is a key regulator within the hematopoietic system and is involved in the regulation of STAT $4 .{ }^{26}$

Despite the differences in allele frequencies of these SNPs between the Caucasian and Asian populations, the risk alleles of all associated SNPs were shared by Caucasians and Asians. In addition, most of the associated SNPs showed comparable effect sizes across ethnic groups. This is also true for the majority of the previously reported SLE risk loci from GWASs for both Caucasians and Asians, such as BLK, IRF5, STAT4, TNFAIP3, TNFSF4 and the HLA region. These SLE GWASs have generally been performed using a similar number of cases and 
controls for both ethnicities, thus differences in the power to detect significant associations for individuals SNPs across ethnicities appears to largely depend on their allele frequencies. These observations underscore the overall genetic homogeneity of SLE.

However, there are differences: for instance, the SNP rs6590330 in the ETS1 locus shows distinct allele frequencies and effect sizes in Caucasians and Asians. Its contribution to the risk of SLE is more pronounced in Asians although it is a disease-associated SNP in both populations. The SNP rs7197475 in the 16p11.2 locus has a low RAF and a large effect in Asians, but it is common in Caucasian populations with a relatively weak contribution to the risk of SLE. Generally the differences in SNP allele frequencies seem to be the major source of genetic heterogeneity rather than the differences in effect sizes. These differences in allele frequencies are most likely a result of genetic drift, but could possibly also be caused by differences in selection due to environmental factors. The present study was performed on 10 Asian SLE SNPs that at the time of initiation of our study were identified as novel compared with the previous GWASs on Caucasians with SLE. Since then, several more studies have been initiated in Asian, which have identified additional loci for SLE. ${ }^{10,12}$ Therefore, a next step will be to perform a more comprehensive independent analysis for population heterogeneity of all published variants associated with SLE in Caucasians and Asians.

In addition, for some of the SNPs we did not detect consistent association signals from all three Caucasian cohorts, which may be due to the differences in RAFs suggested by variable PAR\% across the three Caucasian cohorts. There is always an inherent risk in comparing genotype data from studies performed at different centers. The genotypes for the Swedish and Finnish cohorts were quality controlled together, and the US cohort had previously been extensively QCd as outlined in Gateva et $a l^{7}$ we were not able to apply the same extensive QC to the Swedish and Finnish cohorts as we do not have genome-wide SNP data for these. However, as the genotyping assays used in all studies analyzed here are well validated, and our study focuses on common variation we believe the risk for genotyping errors to be minimal. Thus, we believe that the small differences in allele frequencies we observe between the Caucasian populations are real and not artefacts. Statistical power may be another factor that influences our observations. In the Swedish and the US cohorts, we have reasonable power, whereas the Finnish cohort is smaller in size and provides limited power for any robust conclusion of association for the SNPs without nominally significant $P$-values.

A notable finding in our study was that the TNIP1 gene was nominally associated with SLE in patients with renal or immunological disorder, both in the case-only and case-control subgroup analyses. The association of TNIP1 in the SLE patients with renal and immunological disorder has also been reported in two independent studies on Chinese and Japanese populations. ${ }^{23,24}$ Epidemiological studies have observed higher rates of renal involvement in SLE patients of Asian origin, ${ }^{27}$ which may corroborate our finding that the contribution of TNIP1 to the disease association was 2.1-2.6 times higher in the Asian cohort than the Caucasian cohort. In addition to SLE, association of TNIP1 with psoriasis has been reported for both Caucasian and Asian populations. ${ }^{28,29}$ However, the SNPs reported for psoriasis are only in weak LD with the two TNIP1 SNPs studied here $\left(r^{2} \leqslant 0.11\right.$ in Caucasians and $r^{2} \leqslant 0.18$ in Asians, HapMap release no. 28). TNIP1 has also been found as a risk factor for systemic sclerosis (SSc) in a GWAS based on Caucasian populations. ${ }^{30}$ Interestingly, the TNIP1 SNPs associated with SSc are in medium LD in Caucasians $\left(0.13 \leq r^{2} \leq 0.58\right)$ but in weak LD in Asians $\left(r^{2} \leqslant 0.10\right)$ with the two SLE-associated SNPs in this study. Further studies in patients with SLE and other autoimmune diseases of different ethnical origins and with well-defined clinical characteristics will help to clarify the contribution of TNIP1 to the differences in disease manifestations across populations. The association for SLC15A4 and renal involvement was the only signal to pass multiple testing correction in both the case-only and case-control subphenotype analyses. This has to our knowledge not previously been reported in Caucasians or Asians.

In addition to genetic differences across ethnicities, variation in environment, culture, socioeconomic status and methodological factors could also underlie differences in disease prevalence and manifestations between ethnic groups. ${ }^{27,31}$ Therefore, a multi-ethnic study design is superior to studies based on a single ethnic group, because in the former design the influence of potential non-genetic factors are neutralized, which makes the study more robust in dissecting the impact of genetic factors in complex diseases such as SLE. Here, we have studied common SLE risk variants, which are likely to be shared between different populations; it remains to be seen if rare alleles contribute to risk of SLE in multiple populations in a similar manner.

\section{CONFLICT OF INTEREST}

Dr RR Graham and Dr TW Behrens are full-time employees of Genentech Inc. The remaining authors declare no conflict of interest.

\section{ACKNOWLEDGEMENTS}

SNP genotyping for the Swedish and Finnish SLE case-control cohort was performed at the SNP and SEQ technology platform in Uppsala, Sweden (www.genotyping.se) with the assistance from Dr Sofia Nordman,

Ann-Christin Wiman and Torbjörn Öst. We thank research nurse Rezvan Kiani from the Blood center at Uppsala University Hospital for collecting the patient blood samples. We thank Dr Sari Koskenmies, Dr Taina Hasan and professor Annamari Ranki for their contribution to the recruitment of the Finnish SLE patients. We thank prof Timothy J Vyse and Dr Deborah S Cunninghame Graham for providing data from the UK SLE case-control study. This work was supported by the Knut and Alice Wallenberg Foundation (2011.0073), the Swedish Research Council for Medicine and Health (A0280001, A0258801 and A80741201), the Swedish Research Council for Science and Technology (90559401), the Swedish Rheumatism Association, the Ragnar Söderberg's Foundation, the King Gustaf V 80-year Foundation and COMBINE, the Swedish Heart-Lung Foundation, the Stockholm County Council and Karolinska Institutet (ALF), the Foundation in memory of Clas Groschinsky, and the Swedish Society of Medicine. The UCSF Lupus Genetics Collection for the US SLE case-control cohort was supported by grants from the Alliance for Lupus Research, the Kirkland Scholar Award, and the NIH grants (AR044804, AR02175, AR052300 and M01 RR-000079). The SNP and SEQ Technology Platform, Science for Life Laboratory in Uppsala, is supported by Uppsala University, Uppsala University Hospital and the Swedish Council for Research Infrastructures (80576801 and 70374401).

1 Deng Y, Tsao BP: Genetic susceptibility to systemic lupus erythematosus in the genomic era. Nat Rev Rheumatol 2010; 6: 683-692.

2 Kozyrev SV, Abelson AK, Wojcik J et al: Functional variants in the B-cell gene BANK1 are associated with systemic lupus erythematosus. Nat Genet 2008; 40: 211-216.

3 Hom G, Graham RR, Modrek B et al: Association of systemic lupus erythematosus with C8orf13-BLK and ITGAM-ITGAX. N Engl J Med 2008; 358: 900-909.

4 Harley JB, Alarcon-Riquelme ME, Criswell LA et al: Genome-wide association scan in women with systemic lupus erythematosus identifies susceptibility variants in ITGAM, PXK, KIAA1542 and other loci. Nat Genet 2008; 40: 204-210.

5 Graham RR, Cotsapas C, Davies L et al: Genetic variants near TNFAIP3 on 6q23 are associated with systemic lupus erythematosus. Nat Genet 2008; 40: 1059-1061.

6 Chung SA, Taylor KE, Graham RR et al: Differential genetic associations for systemic lupus erythematosus based on anti-dsDNA autoantibody production. PLoS Genet 2011; 7: e1001323. 
7 Gateva V, Sandling JK, Hom G et al: A large-scale replication study identifies TNIP1, PRDM1, JAZF1, UHRF1BP1 and IL10 as risk loci for systemic lupus erythematosus. Nat Genet 2009; 41: 1228-1233.

8 Harley IT, Kaufman KM, Langefeld CD, Harley JB, Kelly JA: Genetic susceptibility to SLE: new insights from fine mapping and genome-wide association studies. Nat Rev Genet 2009; 10: 285-290.

9 Han JW, Zheng HF, Cui Y et al: Genome-wide association study in a Chinese Han population identifies nine new susceptibility loci for systemic lupus erythematosus. Nat Genet 2009; 41: 1234-1237.

10 Yang W, Shen N, Ye DQ et al: Genome-wide association study in Asian populations identifies variants in ETS1 and WDFY4 associated with systemic lupus erythematosus. PLoS Genet 2010; 6: e1000841.

11 Cunninghame Graham DS, Morris DL, Bhangale TR et al: Association of NCF2, IKZF1, IRF8, IFIH1, and TYK2 with systemic lupus erythematosus. PLoS Genet 2011; 7: e1002341.

12 Okada Y, Shimane K, Kochi Y et al: A genome-wide association study identified aff1 as a susceptibility locus for systemic lupus eyrthematosus in Japanese. PLoS Genet 2012; 8: e1002455.

13 Borchers AT, Naguwa SM, Shoenfeld Y, Gershwin ME: The geoepidemiology of systemic lupus erythematosus. Autoimmun Rev 2010; 9: A277-A287.

14 Plenge RM, Seielstad M, Padyukov L et al: TRAF1-C5 as a risk locus for rheumatoid arthritis-a genomewide study. N Engl J Med 2007; 357: 1199-1209.

15 Koskenmies S, Jarvinen TM, Onkamo P et al: Clinical and laboratory characteristics of Finnish lupus erythematosus patients with cutaneous manifestations. Lupus 2008; 17: 337-347.

16 Koskenmies S, Widen E, Kere J, Julkunen H: Familial systemic lupus erythematosus in Finland. J Rheumatol 2001; 28: 758-760.

17 Tan EM, Cohen AS, Fries JF et al: The 1982 revised criteria for the classification of systemic lupus erythematosus. Arthritis Rheum 1982; 25: 1271-1277.

18 Purcell S, Neale B, Todd-Brown K et al: PLINK: a tool set for whole-genome association and population-based linkage analyses. Am J Hum Genet 2007; 81: 559-575.

19 Higgins JP, Thompson SG, Deeks JJ, Altman DG: Measuring inconsistency in metaanalyses. BMJ 2003; 327: 557-560.

20 Magi R, Morris AP: GWAMA: software for genome-wide association meta-analysis. BMC Bioinformatics 2010; 11: 288.
21 Cole P, MacMahon B: Attributable risk percent in case-control studies. Br J Prev Soc Med 1971; 25: 242-244.

22 Danchenko N, Satia JA, Anthony MS: Epidemiology of systemic lupus erythematosus: a comparison of worldwide disease burden. Lupus 2006; 15: 308-318.

23 Zhong H, Li XL, Li M et al: Replicated associations of TNFAIP3, TNIP1 and ETS1 with systemic lupus erythematosus in a southwestern Chinese population. Arthritis Res Ther 2011; 13: R186.

24 Kawasaki A, Ito S, Furukawa $\mathrm{H}$ et al: Association of TNFAIP3 interacting protein 1, TNIP1 with systemic lupus erythematosus in a Japanese population: a case-control association study. Arthritis Res Ther 2010; 12: R174.

25 Sasawatari S, Okamura T, Kasumi E et al: The solute carrier family 15A4 regulates TLR9 and NOD1 functions in the innate immune system and promotes colitis in mice. Gastroenterology 2011; 140: 1513-1525.

26 Yap WH, Yeoh E, Tay A, Brenner S, Venkatesh B: STAT4 is a target of the hematopoietic zinc-finger transcription factor Ikaros in T cells. FEBS Lett 2005; 579: 4470-4478.

27 Jakes RW, Bae SC, Louthrenoo W, Mok CC, Navarra SV, Kwon N: Systematic review of the epidemiology of systemic lupus erythematosus in the Asia-Pacific region: prevalence, incidence, clinical features, and mortality. Arthritis Care Res 2012; 64: 159-168.

28 Nair RP, Duffin KC, Helms C et al: Genome-wide scan reveals association of psoriasis with IL-23 and NF-kappaB pathways. Nat Genet 2009; 41: 199-204.

29 Sun LD, Cheng H, Wang ZX et al: Association analyses identify six new psoriasis susceptibility loci in the Chinese population. Nat Genet 2010; 42: 1005-1009.

30 Allanore Y, Saad M, Dieude P et al: Genome-wide scan identifies TNIP1, PSORS1C1, and RHOB as novel risk loci for systemic sclerosis. PLoS Genet 2011; 7: e1002091.

31 Osio-Salido E, Manapat-Reyes H: Epidemiology of systemic lupus erythematosus in Asia. Lupus 2010; 19: 1365-1373.

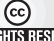

This work is licensed under the Creative Commons Attribution-NonCommercial-No Derivative Works 3.0 Unported License. To view a copy of this license, visit http://creativecommons.org/licenses/by-nc-nd/3.0/

Supplementary Information accompanies the paper on European Journal of Human Genetics website (http://www.nature.com/ejhg) 